\title{
Decision-Directed Fine Synchronization in OFDM Systems
}

\author{
Kai Shi, Erchin Serpedin, and Philippe Ciblat
}

\begin{abstract}
A new decision-directed (DD) synchronization scheme is proposed for joint estimation of carrier frequency offset (CFO) and sampling clock frequency offset (SFO) in orthogonal frequency-division multiplexing (OFDM) systems. By exploiting the hard decisions, we report accurate estimators of residual CFO and small SFO. The performance analysis and simulation results indicate that the proposed novel DD scheme achieves much better performance than the conventional pilot-based schemes in both additive white Gaussian noise and frequency-selective channels.
\end{abstract}

Index Terms-Carrier frequency offset (CFO), fast Fourier transform (FFT), orthogonal frequency-division multiplexing (OFDM), sampling clock frequency offset (SFO), synchronization.

\section{INTRODUCTION}

$\mathbf{T}$ HE orthogonal frequency-division multiplexing (OFDM) system is well fit for high-speed transmissions in highly frequency-selective (F-S) channels. However, OFDM is sensitive to synchronization errors. Numerous papers [1]-[4] deal with coarse frame (timing) and carrier frequency offset (CFO) synchronization, and most of them [2]-[4] proposed estimators before the fast Fourier transform (FFT). However, after coarse synchronization, there might still be a residual CFO and a sampling clock frequency offset (SFO), which will introduce time and subcarrier varying phase rotations [5], [6] and FFT window shift.

To remove the effect of CFO and SFO, some authors proposed pilot-based post-FFT synchronizers [7]-[10]. Although the estimator [7] appears to work under general channel conditions, no analytical result has been reported to assess its unbiasedness in F-S channels. Also, the alternative estimator [11] appears to be biased in F-S channels. In this letter, we propose a new decision-directed (DD) post-FFT CFO and SFO synchronization scheme without relying on pilots. It is shown that the proposed $\mathrm{CFO}$ and SFO estimators are (approximately) unbiased in both additive white Gaussian noise (AWGN) as well as F-S channels. Analytical closed-form expressions of the mean-square error (MSE) of the proposed estimators are also reported for AWGN channels.

Paper approved by R. De Gaudenzi, the Editor for Synchronization and CDMA of the IEEE Communications Society. Manuscript received September 24, 2003; revised March 10, 2004 and October 11, 2004. This work was supported by the National Science Foundation under Award CCR-0092901, in part by the ARP Program, and in part by the TITF Program of Texas A\&M University. This paper was presented in part at the 2004 International Conference on Acoustics, Speech, and Signal Processing.

K. Shi and E. Serpedin are with the Department of Electrical Engineering, Texas A\&M University, College Station, TX 77843-3128 USA (e-mail: serpedin@ee.tamu.edu).

P. Ciblat is with the Departement Communications et Electronique, ENST, F-75013 Paris, France.

Digital Object Identifier 10.1109/TCOMM.2005.843463

\section{Signal Models}

The transmitted complex baseband OFDM signal can be described by

$$
s(t)=\frac{1}{\sqrt{T_{u}}} \sum_{l=0}^{\infty} \sum_{k=-K / 2, k \neq 0}^{K / 2} a_{l, k} e^{j 2 \pi\left(k / T_{u}\right)\left(t-T_{g}-l T\right)} g(t-l T)
$$

where $a_{l, k}$ denotes the complex data modulated on the $f_{k}=$ $k / T_{u}$ subcarrier frequency of the $l_{t h}$ OFDM symbol with the unit variance, $g(t)$ is a rectangular pulse with unit amplitude during $0 \leq t<T$, and $T_{g}$ is the length of cyclic prefix. A discrete-time implementation (with the sampling period $T_{s}=$ $\left.T_{u} / N\right)$ of $s(t)$ is generated by means of an $N$-point inverse fast Fourier transform (IFFT). In addition, $K$ is chosen to be less than $N$ to avoid spectrum aliasing. Therefore, the symbol period is $T=T_{g}+T_{u}$, which corresponds to $M=N+N_{g}$ samples.

The received signal sampled with the period $T_{s}^{\prime}$, in the presence of CFO $f_{e}$, timing offset $n_{e} T_{s}^{\prime}$ and small SFO $\epsilon=\left(T_{s}^{\prime}-\right.$ $\left.T_{s}\right) / T_{s}$, is given by

$r\left(n T_{s}^{\prime}\right)=e^{j 2 \pi f_{e} n T_{s}^{\prime}} \sum_{i} h_{i}\left(n T_{s}^{\prime}\right) s\left(n T_{s}^{\prime}-\tau_{i}-n_{e} T_{s}^{\prime}\right)+w\left(n T_{s}^{\prime}\right)$

where $\left\{h_{i}\left(n T_{s}^{\prime}\right)\right\}$ present the samples of channel. We assume that the channel presents normalized power $\sum_{i} E\left[\left|h_{i}\left(n T_{s}^{\prime}\right)\right|^{2}\right]=1$, and its maximum delay spread $\tau_{\max }$ is less than $T_{g}$. In addition, $w\left(n T_{s}^{\prime}\right)$ denotes complex AWGN with variance $\sigma_{n}^{2}=E\left\{\left|w\left(n T_{s}^{\prime}\right)\right|^{2}\right\}$. The average signal-to-noise ratio (SNR) for data subcarriers is defined as $E_{s} / N_{0}=1 / \sigma_{n}^{2}$

After the coarse timing estimation, $\hat{n}_{e}$ is used by the FFT window controller. Therefore, the FFT window can be assumed to start from the intersymbol interference (ISI)-free area $\left[\tau_{\max }+\right.$ $\left.1+l M, N_{g}+l M\right] T_{s}^{\prime}$. To reduce possible intercarrier interference (ICI), a coarse CFO estimate $\hat{f}_{e}$ is used by the frequency-corrector block. The channel is assumed to be constant during an OFDM symbol duration, and its Fourier transform is given by $H_{l, k}=\sum_{n=0}^{\tau_{\max }} h_{l, n} e^{-j 2 \pi k(n / N)}, h_{l, n}=h_{n}\left(l M T_{s}^{\prime}\right)$.

The output of the $N$-point FFT block can be expressed as $z_{l, k}=\sum_{n=0}^{N-1} r_{l, n} e^{-j 2 \pi k n / N} / \sqrt{N}$, where $r_{l, n}=r\left(\left(n+N_{g}+\right.\right.$ $l M) T_{s}^{\prime}$ ). Taking into account the small SFO $\epsilon$ and residual CFO $f_{N}=f_{e} T_{u}[6]$, the $l_{t h}$ symbol of the $N$-point FFT block takes the expression

$$
z_{l, k} \approx a_{l, k} H_{l, k} e^{j \pi \theta_{k}(N-1) / N} e^{j 2 \pi \theta_{k}\left(N_{g}+l M\right) / N}+\mathrm{ICI}+n_{l, k}
$$

where $n_{l, k}$ has the same characteristic as $w\left(n T_{s}^{\prime}\right)$ and $\theta_{k}=$ $f_{N}(1+\epsilon)+\epsilon k \approx f_{N}+\epsilon k$. In the approximate expression (3), the ICI caused by small CFO and SFO can be omitted, since 


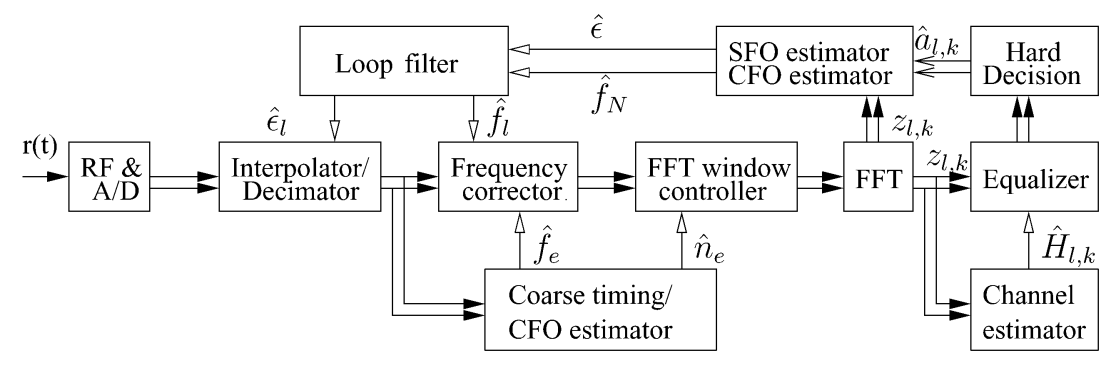

Fig. 1. Receiver structure.

its power is very small compared with the additive noise $\left(n_{l, k}\right)$ power [5], [6]. The effect of slow drifts of the FFT window caused by small SFO $\epsilon$ is not shown in (3).

Some phase-compensation techniques, e.g., [12], can be used to remove the phase rotation (3), and such techniques are popular in short packet transmission systems. However, the window shift caused by the small SFO $\epsilon$, can be very large, enough to introduce intolerable ISI in long packet-based transmissions systems. For example, multiband-OFDM proposal [13] can have up to 1000 symbols per packet, and the window shift caused by a $40 \mathrm{ppm}$ SFO can be up to six samples. Thus, to remove both phase rotation and window shift, we need to estimate $f_{N}$ and $\epsilon$.

Similar to [6], we take the conjugate product of two consecutive OFDM symbols

$$
x_{l, k}=z_{l, k} \cdot z_{l-1, k}^{*} \approx e^{j 2 \pi \rho \theta_{k}} a_{l, k} a_{l-1, k}^{*}\left|H_{l, k}\right|^{2}+\text { noise }
$$

where $\rho=M / N$, * denotes the conjugate operation, and we assume $H_{l, k} \approx H_{l-1, k}$.

\section{DATA-AidED ESTIMATOR}

A post-FFT data-aided (DA) CFO and SFO estimator was proposed in [7] and [8]

$$
\hat{f}_{N}=\frac{1}{2 \pi \rho} \cdot \frac{\varphi_{l, 1}+\varphi_{l, 2}}{2}, \quad \hat{\epsilon}=\frac{1}{2 \pi \rho} \cdot \frac{\varphi_{l, 2}-\varphi_{l, 1}}{\frac{K}{2}}
$$

where $\varphi_{l,(1 \mid 2)}=\arg \left[\sum_{k \in P_{(1 \mid 2)}} x_{l, k}\right], P_{(1 \mid 2)}$ denotes the first and the second half of pilots ( $M$-ary phase-shift keying (MPSK) modulated), respectively, which are assumed symmetrically and uniformly distributed around DC $(k=0)$.

It is easy to prove that estimators (5) are unbiased in the presence of AWGN and flat-fading channels. In [8], the MSE of (5) in AWGN channels are reported

$$
\begin{aligned}
\operatorname{MSE}\left(\hat{f}_{N}\right) & =\frac{1}{4 \pi^{2} \rho^{2} N_{P} \cdot \frac{E_{s}}{N_{0}}} \\
\operatorname{MSE}(\hat{\epsilon}) & =\frac{4}{\pi^{2} \rho^{2} K^{2} N_{P} \cdot \frac{E_{s}}{N_{0}}}
\end{aligned}
$$

respectively, where $N_{P}$ stands for the number of pilots per symbol. To reduce synchronization overhead, we may use a reduced number of pilots. However, it will degrade the performance in (6). Furthermore, the pilot-based approach may be not possible in noncoherent systems since there is no pilot available.
In fact, the above estimator is not the optimal one. We find that the CFO estimator [14] for single-carrier systems can be easily modified to estimate SFO in OFDM systems and presents slightly better performance (not reported here due to space limitations). However, due to the high complexity of estimator in [14], we will focus on estimators similar to (5) hereafter.

\section{Proposed SynChronizATION SCHEME}

\section{A. DD Estimator}

In this paper, we propose a DD scheme to replace the conventional DA scheme

$$
\hat{f}_{N}=\frac{1}{2 \pi \rho} \cdot \frac{\varphi_{l, 1}^{\prime}+\varphi^{\prime}{ }_{l, 2}}{2}, \quad \hat{\epsilon}=\frac{1}{2 \pi \rho} \cdot \frac{\varphi_{l, 2}^{\prime}-\varphi^{\prime}{ }_{l, 1}}{\frac{K}{2}+1}
$$

where

$$
\varphi_{l,(1 \mid 2)}^{\prime}=\arg \left[A_{l,(1 \mid 2)}\right], \quad A_{l,(1 \mid 2)}=\sum_{k \in C_{(1 \mid 2)}} \frac{z_{l, k} z_{l-1, k}^{*}}{\hat{a}_{l, k} \hat{a}_{l-1, k}^{*}}
$$

and $C_{1}=[-K / 2,-1], C_{2}=[1, K / 2]$ denote the first and second half of data subcarriers, respectively. For coherent systems, data decisions are available right after the coarse synchronization and channel estimation. For noncoherent systems, we can use data decisions right after coarse synchronization.

To obtain reliable $\hat{a}_{l, k}$, we may use the outer channel decoder output to reconstruct the complex $\hat{a}_{l, k}$. However, such an approach requires more memory and arithmetic operations. Furthermore, the decoding and interleaver delay will degrade the tracking performance [16, pp. 239)].

A simpler way is to use the hard data decisions. In highly F-S channels, the data decisions in spectral nulls might be unreliable. However, these decision errors have small contribution on the final estimate since $\left|H_{l, k}\right|^{2}$ are very small (4), and their effects are averaged out in (7) and (8). The decision directed synchronizer is plotted in Fig. 1.

In Appendix A, we show that the MSE of the proposed estimator in AWGN channels is given by

$$
\begin{aligned}
\operatorname{MSE}\left(\hat{f}_{N}\right) & =\frac{1}{4 \pi^{2} \rho^{2} K \cdot \frac{E_{s}}{N_{0}}} \\
\operatorname{MSE}(\hat{\epsilon}) & =\frac{4}{\pi^{2} \rho^{2} K(K+2)^{2} \cdot \frac{E_{s}}{N_{0}}}
\end{aligned}
$$

which are much smaller than (5). Due to channel fading effects, as shown by the computer simulations in Fig. 2, the MSE performance in F-S channels is slightly worse than (9). 


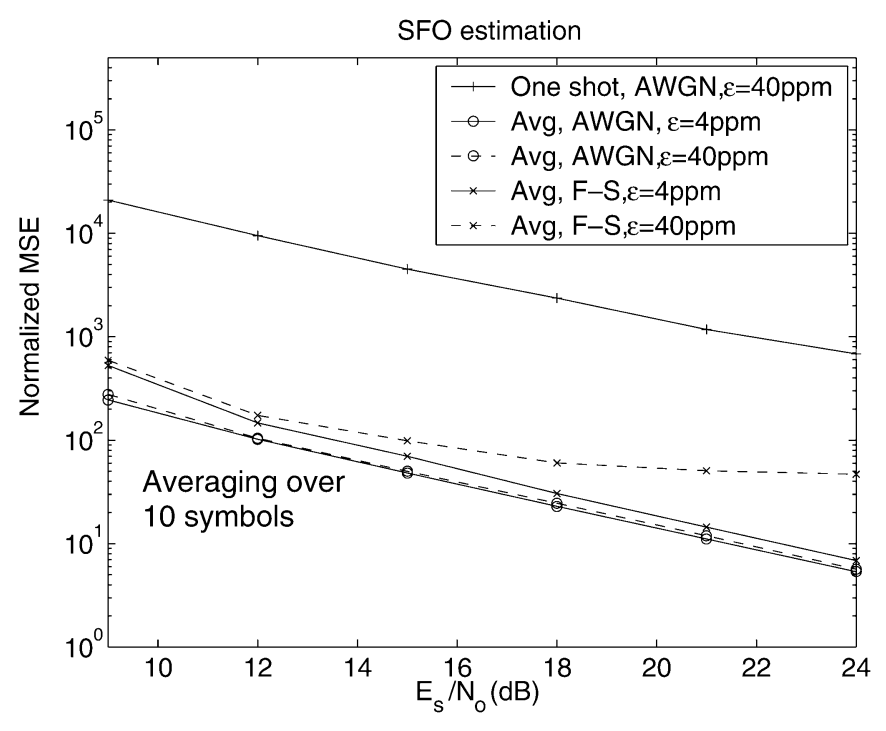

Fig. 2. Normalized MSE (normalized by $10^{-12}$ ) of open-loop DD SFO estimators.

In Appendix B, we have also shown that estimators (7) are approximately unbiased in F-S channels for small $\epsilon$. A similar proof can be carried out for the estimator (5) using some slight modifications. As we will show later in simulations, the above one-shot estimation may be not accurate enough for correction. As shown in Fig. 2, averaging over 10 symbols can greatly decrease the MSE in AWGN channels. Unfortunately, in F-S channels, an error floor is found for large SFO $\epsilon$, which can be explained that (5) and (7) are not unbiased anymore for large SFO.

\section{B. Closed-Loop Scheme}

The above results suggest that we use a closed-loop synchronization scheme. The one-shot estimates are postprocessed by the following first-order tracking loop filters:

$$
\hat{f}_{l}=\hat{f}_{l-1}+\gamma_{f} \hat{f}_{N}, \quad \hat{\epsilon}_{l}=\hat{\epsilon}_{l-1}+\gamma_{\epsilon} \hat{\epsilon}
$$

Symbol by symbol, the above loop filters update the control parameters of number-controlled oscillators in the interpolator and frequency corrector, and the residual frequency errors gradually converge to smaller values.

After convergence, the estimators exhibit small fluctuations about the stable equilibrium points. Based on a linearized equivalent model [15], we can derive the tracking performance as follows

$$
\mathrm{MSE}=\int_{-1 /(2 T)}^{1 /(2 T)} S(f)|G(f)|^{2} d f
$$

where $S(f)$ is the power spectral density (PSD) of loop noise (derived in Appendix A)

$$
\begin{aligned}
& S_{\mathrm{CFO}}(f)=\frac{\sigma_{n}^{2} T[1-\cos (2 \pi f T)]}{4 \pi^{2} \rho^{2} K} \\
& S_{\mathrm{SFO}}(f)=\frac{4 \sigma_{n}^{2} T[1-\cos (2 \pi f T)]}{\pi^{2} \rho^{2} K(K+2)^{2}}
\end{aligned}
$$

and $G(f)$ is the closed-loop transfer function given by

$$
G(f)=\frac{-\gamma}{e^{j 2 \pi f T}-(1-\gamma)}
$$

Substituting (12)-(13) into (11), we can easily find the MSE of closed-loop DD estimators

$$
\begin{aligned}
\operatorname{MSE}\left(\hat{f}_{l}\right) & =\frac{\frac{\gamma_{f}^{2}}{\left(2-\gamma_{f}\right)}}{4 \pi^{2} \rho^{2} K \cdot \frac{E_{s}}{N_{0}}} \\
\operatorname{MSE}\left(\hat{\epsilon}_{l}\right) & =\frac{\frac{4 \gamma_{\epsilon}^{2}}{\left(2-\gamma_{\epsilon}\right)}}{\pi^{2} \rho^{2} K(K+2)^{2} \cdot \frac{E_{s}}{N_{0}}} .
\end{aligned}
$$

Similarly, we obtain the MSE of closed-loop DA estimators in [7] and [8]

$$
\begin{aligned}
\operatorname{MSE}\left(\hat{f}_{l}\right) & =\frac{\frac{\gamma_{f}^{2}}{\left(2-\gamma_{f}\right)}}{4 \pi^{2} \rho^{2} N_{P} \cdot \frac{E_{s}}{N_{0}}} \\
\operatorname{MSE}\left(\hat{\epsilon}_{l}\right) & =\frac{\frac{4 \gamma_{\epsilon}^{2}}{\left(2-\gamma_{\epsilon}\right)}}{\pi^{2} \rho^{2} K^{2} N_{P} \cdot \frac{E_{s}}{N_{0}}} .
\end{aligned}
$$

\section{COMPUTER Simulations}

In our simulations, we assume an OFDM system similar to [13] with $N=128$ subcarriers and guard interval of 32 . There are 10 pilot subcarriers inserted into 120 quaternary phase-shift keying (QPSK) data-modulated subcarriers in the DA scheme, while no pilot is inserted in the DD scheme. We assume a 12-path F-S channel with exponentially decaying power profile and the root-mean square delay spread is $1.25 T_{s}$. A rate-3/4 (punctured from 1/2) convolutional encoder with generator polynomial (133 171) and a block interleaver $(16 \times 15)$ are used in the system. The simulation results are obtained using 2000 Monte Carlo trials for each SNR value. The SFO $\epsilon$ and CFO $f_{N}$ are assumed equal to $40 \mathrm{ppm}$ and $0.6 \%$, respectively. For simplicity, we assume the channel static during the whole packet (150 symbols), and the first two symbols are used for one-shot DA channel estimation. Due to space limitations, the simulation results provided below are mainly for SFO estimation.

From [17], the modified Cramer-Rao bound (MCRB) for SFO estimation in AWGN channels is given by

$$
\operatorname{MCRB}(\epsilon) \approx \frac{9}{2 \pi^{2} K^{3} L^{3}\left(\frac{M}{N}\right)^{2} \frac{E_{s}}{N_{0}}}
$$

where $L$ is the equivalent number of symbols $L=\sqrt{2-\gamma_{\epsilon}} / \gamma_{\epsilon}$. In the loop filters, we use $\gamma_{\epsilon}=0.2$ and $\gamma_{f}=0.3$ for both DD and DA schemes. The closed-loop tracking performance of the DD scheme in Fig. 3, which is much closer to the MCRB than the conventional DA scheme, corroborates the closed-loop analysis result presented in Section IV. At low SNRs, the simulation results for the DD scheme deviate from the analysis results, a fact which is due to error propagations. However, error propagations are hardly found for SNRs larger than $12 \mathrm{~dB}$. 


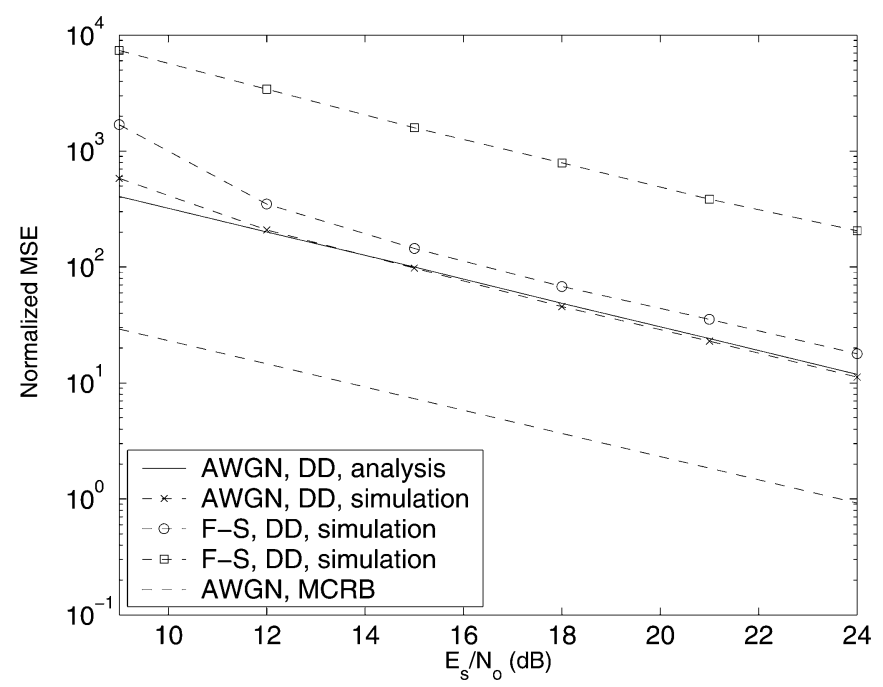

Fig. 3. Normalized MSE (normalized by $10^{-12}$ ) of closed-loop SFO estimators.

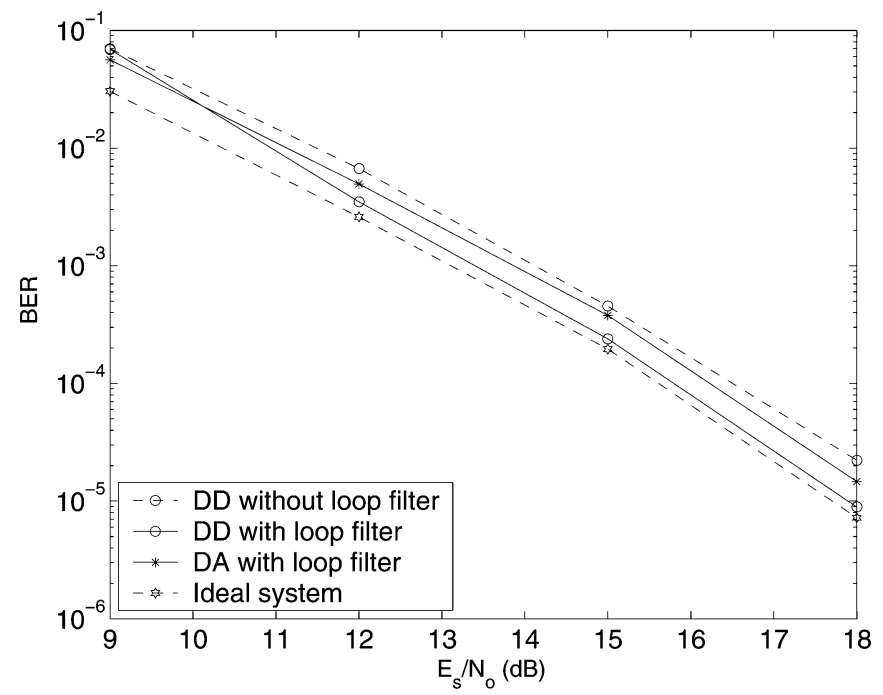

Fig. 4. BER performance comparison.

In Fig. 4, we plot the bit-error rate (BER) performance for different systems. The BER performance of the system using the DD scheme with loop filter is very close to that of the ideal system which assumes perfect synchronization. However, $0.7 \mathrm{~dB}$ performance degradation will be introduced if the loop filter is not used. Compared with the DD scheme with the same loop filter, the DA scheme presents $0.5 \mathrm{~dB}$ performance degradation.

\section{CONCLUSIONS}

This letter has introduced a new DD post-FFT joint estimator for the CFO and SFO in OFDM systems. By performance analysis and computer simulations, we prove that our new scheme exhibits much better performance, compared with the conventional DA scheme in both AWGN and F-S channels. Since we save the pilots for synchronization, the throughput of the system is also increased. With very little additional hardware, this new synchronization scheme can be implemented in many wireless OFDM systems.

\section{APPENDIX A}

\section{Performance AnAlysis in AWGN Channels}

For simplicity, MPSK modulation is assumed in the following analysis. In AWGN channels, assuming correct decisions and substituting (3) into (8), one can find

$$
A_{l, k}=z_{l, k} a_{l, k}^{*} z_{l-1, k}^{*} a_{l-1, k} \approx e^{j 2 \pi \rho \theta_{k}}+v_{l, k}
$$

where $v_{l, k}=n_{l, k} a_{l, k}^{*} e^{-j \phi_{l-1, k}}+n_{l-1, k}^{*} a_{l-1, k} e^{j \phi_{l, k}}$, and the products of two noise terms are negligible. From (7), denoting $\alpha=e^{-j 2 \pi \rho f_{N}}$ and making use of $\tan (a \pm b) \approx \tan (a) \pm \tan (b)$ (if $\tan (a) \tan (b) \ll 1$ ), we obtain

$$
\begin{aligned}
\tan \left[4 \pi \rho\left(\hat{f}_{N}-f_{N}\right)\right] & \approx \frac{\Im\left(A_{l, 1} \alpha\right)}{\Re\left(A_{l, 1} \alpha\right)}+\frac{\Im\left(A_{l, 2} \alpha\right)}{\Re\left(A_{l, 2} \alpha\right)} \\
& \approx 4 \pi \rho\left(\hat{f}_{N}-f_{N}\right)
\end{aligned}
$$

where $\Re(x)$ and $\Im(x)$ denote the real and imaginary parts of $x$, respectively. Substituting (17) into (8) and (18), it follows that

$$
\begin{aligned}
e_{l} & \approx \frac{1}{4 \pi \rho}\left[\frac{\Im\left(A_{l, 1} \alpha\right)}{\sum_{k \in C_{1}} \cos (\pi \rho \epsilon k)}+\frac{\Im\left(A_{l, 2} \alpha\right)}{\sum_{k \in C_{2}} \cos (\pi \rho \epsilon k)}\right] \\
& \approx \frac{1}{2 \pi \rho K} \Im\left[\alpha \sum_{k \in C_{1}, C_{2}} v_{l, k}\right]
\end{aligned}
$$

where we assume $\cos (\pi \rho \epsilon k) \approx 1$ and $\sin (\pi \rho \epsilon k) \approx 0$ for small SFO $\epsilon$. The MSE of CFO estimator can be obtained after some straightforward calculations

$$
\operatorname{MSE}\left(\hat{f}_{N}\right)=\frac{1}{4 \pi^{2} \rho^{2} K \cdot \frac{E_{s}}{N_{0}}}
$$

Similarly, one can find the MSE of SFO estimator

$$
\operatorname{MSE}(\epsilon)=\frac{4}{\pi^{2} \rho^{2} K(K+2)^{2} \cdot \frac{E_{s}}{N_{0}}}
$$

From (19), after some manipulations, one can find that the CFO estimation noise $e_{l}=\hat{f}_{N}-f_{N}$ has the following autocorrelation:

$$
R_{e}(m):=E\left\{e_{l} e_{l+m}\right\}= \begin{cases}\frac{\sigma_{n}^{2}}{4 \pi^{2} \rho^{2} K}, & m=0 \\ -\frac{\sigma_{n}^{2}}{8 \pi^{2} \rho^{2} K}, & m= \pm 1 \\ 0, & \text { otherwise }\end{cases}
$$

Thus, the PSD of CFO estimation noise is given by

$S_{\mathrm{CFO}}(f)=T \sum_{m} R_{e}(m) e^{-j 2 \pi m f T}=\frac{\sigma_{n}^{2} T[1-\cos (2 \pi f T)]}{4 \pi^{2} \rho^{2} K}$.

Similarly, the PSD of SFO estimation noise can be obtained

$$
S_{\mathrm{SFO}}(f)=\frac{4 \sigma_{n}^{2} T[1-\cos (2 \pi f T)]}{\pi^{2} \rho^{2} K(K+2)^{2}}
$$




\section{APPENDIX B \\ UNBIASEDNESS OF ESTIMATOR (7) IN \\ FREQUENCY-SELECTIVE CHANNELS}

Assuming correct decisions $\hat{a}_{l, k}$ in (8), we obtain

$$
A_{l, 1}=\sum_{k \in C_{1}}\left|H_{l, k}\right|^{2} e^{j 2 \pi \rho \theta_{k}}+\sum_{k \in C_{1}} v_{l, k}
$$

where $v_{l, k}=n_{l, k} a_{l, k}^{*} H_{l-1, k}^{*} e^{-j \phi_{l-1, k}}+n_{l-1, k}^{*} a_{l-1, k}$ $H_{l, k} e^{j \phi_{l, k}}$.

Thus, the first term of the right-hand side of $A_{l, 1}$ can be rewritten as

$$
\begin{aligned}
A_{l, s 1}= & e^{j 2 \pi \rho f_{N}} \\
& \times\left(e^{-j \pi \rho \epsilon(K / 2+1)} S i(\epsilon, K) \sum_{n=0}^{\tau_{\max }}\left|h_{l, n}\right|^{2}\right. \\
& \left.\quad+\sum_{n=0}^{\tau_{\max }} \sum_{m=n+1}^{\tau_{\max }} \sum_{k \in C_{1}} 2 \Re\left\{\Gamma_{l, n, m, k}\right\} e^{j 2 \pi \rho \epsilon k}\right)
\end{aligned}
$$

where $\operatorname{Si}(\epsilon, K)=(\sin (\pi \rho \epsilon K / 2) / \sin (\pi \rho \epsilon)) \approx K / 2$ and $\Gamma_{l, n, m, k}=h_{l, n} h_{l, m}^{*} e^{-j 2 \pi k(n-m) / N}$.

Defining $\Delta=n-m$, we obtain

$$
\begin{aligned}
& \sum_{k \in C_{1}} 2 \Re\left\{\Gamma_{l, n, m, k}\right\} e^{j 2 \pi \rho \epsilon k} \\
&=h_{l, n} h_{l, m}^{*} e^{-j \pi(\rho \epsilon-\Delta / N)(K / 2+1)} \frac{\sin \left[\frac{\pi K\left(\frac{\rho \epsilon-\Delta}{N}\right)}{2}\right]}{\sin \left[\pi\left(\frac{\rho \epsilon-\Delta}{N}\right)\right]} \\
&+h_{l, n}^{*} h_{l, m} e^{-j \pi(\rho \epsilon+\Delta / N)(K / 2+1)} \frac{\sin \left[\frac{\pi K\left(\frac{\rho \epsilon+\Delta}{N}\right)}{2}\right]}{\sin \left[\pi\left(\frac{\rho \epsilon+\Delta}{N}\right)\right]} .
\end{aligned}
$$

Thanks to the approximation $(\sin [\pi K(\rho \epsilon \pm \Delta / N) / 2] /$ $\sin [\pi(\rho \epsilon \pm \Delta / N)]) \approx(\sin [\pi \Delta K /(2 N)] / \sin (\pi \Delta / N))$, which is valid if $\epsilon \ll \Delta / N$, ${ }^{1}$ we can approximate (27) as

$\sum_{k \in C_{1}} 2 \Re\left\{\Gamma_{l, n, m, k}\right\} e^{j 2 \pi \rho \epsilon k} \approx e^{-j \pi \rho \epsilon(K / 2+1)} \sum_{k \in C_{1}} 2 \Re\left\{\Gamma_{l, n, m, k}\right\}$

\footnotetext{
${ }^{1}$ The accuracy of approximation depends on the value of $\epsilon$. For large $\epsilon$, the approximation is not accurate anymore and the unbiasedness of (7) can not be shown along this line of proof.
}

and rewrite $A_{l, 1}$ as

$$
A_{l, 1} \approx e^{j 2 \pi \rho f_{N}} e^{-j \pi \rho \epsilon(K / 2+1)} \sum_{k \in C_{1}}\left|H_{l, k}\right|^{2}+\sum_{k \in C_{1}} v_{l, k}
$$

Similarly, we can make the following approximation:

$$
A_{l, 2} \approx e^{j 2 \pi \rho f_{N}} e^{j \pi \rho \epsilon(K / 2+1)} \sum_{k \in C_{2}}\left|H_{l, k}\right|^{2}+\sum_{k \in C_{2}} v_{l, k}
$$

Substituting (29)-(30) into (7), it is easy to find that (7) is approximately unbiased for slow-fading F-S channels.

\section{REFERENCES}

[1] P. H. Moose, "A technique for orthogonal frequency division multiplexing frequency offset correction," IEEE Trans. Commun., vol. 42, pp. 2908-2914, Oct. 1994.

[2] T. M. Schmidl and D. C. Cox, "Robust frequency and timing synchronization for OFDM," IEEE Trans. Commun., vol. 45, pp. 1613-1621, Dec. 1997.

[3] J. J. van de Beek, M. Sandell, and P. O. Borjesson, "ML estimation of time and frequency offset in OFDM systems," IEEE Trans. Signal Process., vol. 45, pp. 1800-1805, Jul. 1997.

[4] K. Shi and E. Serpedin, "Coarse frame and carrier synchronization for OFDM systems: A new metric and performance evaluation," IEEE Trans. Wireless Commun., vol. 3, pp. 1271-1284, Jul. 2004, to be published.

[5] T. Pollet et al., "The BER performance of OFDM systems using nonsynchronized sampling," in Proc. GLOBECOM, 1994, pp. 253-257.

[6] M. Speth, S. A. Fechtel, G. Fock, and H. Meyr, "Optimum receiver design for wireless broadband systems using OFDM-Part I," IEEE Trans. Commun., vol. 47, pp. 1668-1677, Nov. 1999.

[7] _ , "Optimum receiver design for wireless broadband systems using OFDM-Part II," IEEE Trans. Commun., vol. 49, pp. 571-578, Apr. 2001.

[8] S. A. Fechtel, "OFDM carrier and sampling frequency synchronization and its performance on stationary and mobile channels," IEEE Trans. Consum. Electron., vol. 46, pp. 438-441, Aug. 2000.

[9] K. K. Dong, H. D. Sang, C. B. Hong, C. J. Hyung, and K. B. Ki, “A new joint algorithm of symbol timing recovery and sampling clock adjustment for OFDM systems," IEEE Trans. Consum. Electron., vol. 44, pp. 1142-1149, Aug. 1998.

[10] M. J. F.-G. Garcia and J. M. Paez-Borrallo, "Tracking of time misalignments for OFDM systems in multipath fading channels," IEEE Trans. Consum. Electron., vol. 48, pp. 982-989, Nov. 2002.

[11] B. Yang, K. B. Letaief, R. S. Cheng, and Z. Cao, "Timing recovery for OFDM transmission," IEEE J. Sel. Areas Commun., vol. 18, pp. 2278-2290, Nov. 2000.

[12] K. Nikitopoulos and A. Polydoros, "Compensation schemes for phase noise and residual frequency offset in OFDM systems," in Proc. IEEE GLOBECOM, Nov. 2001, pp. 25-29.

[13] Physical Layer Submission to 802.15 Task Group 3a: Multiband Orthog onal Frequency-Division Multiplexing, IEEE 802.15-03/268r0, 2003.

[14] M. Luise and R. Reggiannini, "Carrier frequency recovery in all-digital modems for burst-mode transmissions," IEEE Trans. Commun., vol. 43, pp. 1169-1178, Feb.-Apr. 1995.

[15] H. Meyr et al., Digital Communication Receivers: Synchronization, Channel Estimation and Signal Processing. New York: Wiley, 1998.

[16] U. Mengali and A. N. D'Andrea, Synchronization Techniques for Digital Receivers. New York: Plenum, 1997.

[17] K. Shi and E. Serpedin, "On the estimation of sampling clock frequency offset in single and multiple carrier systems," in Proc. CISS, Princeton, NJ, Mar. 17-19, 2004, pp. 233-238. 